\title{
"I love my ECPs": challenges to bridging emergency contraceptive users to more effective contraceptive methods in Ghana
}

\author{
Kelly Ladin L'Engle, ${ }^{1}$ Laura Hinson, ${ }^{2}$ Dawn Chin-Quee ${ }^{3}$
}

'Behavioral and Social Sciences, FHI, Durham, NC, USA 2Department of Population, Family, and Reproductive Health, Johns Hopkins Bloomberg School of Public Health, Baltimore, MD, USA

${ }^{3}$ Program Sciences, FHI, Durham, NC, USA

\section{Correspondence to} Dr Kelly Ladin L'Engle, FHI, PO Box 13950, Research Triangle Park, NC 27709, USA; klengle@fhi.org

Received 24 March 2010 Accepted 14 December 2010 Published Online First 17 April 2011

\begin{abstract}
Background and methods Emergency

contraceptive pills (ECPs) are becoming more popular, yet little is known about the contraceptive preferences of women who take ECPs. Women purchasing ECPs were recruited from pharmacies in Accra, Ghana. A total of 24 semi-structured, qualitative interviews were conducted in May 2008. Results Nearly all participants preferred ECPs to other contraceptive methods. Although fear of side effects from oral contraceptive pills (OCPs), intrauterine devices and injectables were deterrents to use of those methods, side effects from ECPs were acceptable to this small and highly self-selected group of ECP users. Participants had little knowledge about how other contraceptive methods work and expressed a strong distrust and dislike of condoms.

Discussion and conclusion Study participants loved their ECPs, despite minor discomforts like bleeding, and most had no concerns about repeated use, though these findings may not apply to women outside Accra or women who obtain ECPs from non-pharmacy settings. Future interventions should work to dispel myths about OCPs, condoms and other modern methods, and focus on basic contraception education.
\end{abstract}

Emergency contraceptive pills (ECPs) represent a small but growing segment of the contraceptive market. Recent availability of emergency contraception (EC) products in the private sector has fuelled interest in ECPs as an important contraceptive option for women. ECPs are currently underutilised, ${ }^{12}$ but private sector promotion in pharmacy settings, coupled with preliminary data indicating increased demand, suggest that ECPs will become increasingly important as an alternative contraceptive option for women and couples. ${ }^{3-5}$

ECPs are recommended to avoid unwanted pregnancy when no contraceptive has

\section{Key message points}

Among pharmacy-based emergency contraceptive pill (ECP) users, emergency contraception was well-liked, occupied an important role in the contraceptive method mix, and was often preferred to other types of modern contraception.

- Women who use ECPs may benefit from additional education about the full range of contraceptive methods.

been used, when there is a contraceptive failure or incorrect use, or in cases of sexual assault. ${ }^{6}$ Although recommendations do not enumerate how many times ECPs can or should be used in a specific time frame, global media reports have focused attention on repeated use of ECPs, implying intentional and irresponsible use of this postcoital method. For example, a major Jamaican newspaper reported that pharmacists were concerned that women were using ECPs in place of condoms and more effective long-term methods of contraception (henceforth referred to as 'regular' contraceptives). ${ }^{7}$ Media in Nairobi, Kenya warned that women were "taking ECPs like chocolate". ${ }^{8}$ While ECPs can safely be taken as many times as necessary, ${ }^{9}$ little has been documented on why women choose ECPs over other methods in nonemergency use situations.

Importantly, contraceptive options that are more effective, have fewer side effects, and cost less than ECPs - methods such as oral contraceptive pills (OCPs), intrauterine devices (IUDs) and depot medroxyprogesterone acetate (DMPA) $\left(\right.$ Depo-Provera $\left.^{\circledR}\right)$ - are available for women who need longer-term protection from pregnancy. Although it is difficult to compare effectiveness rates between ECPs and other contraceptive methods, 'regular' contraceptive methods are available that, when used correctly, provide highly effective, longer-term pregnancy prevention. ${ }^{10}$ In addition, ECPs cause more side 
effects than other hormonal contraceptive methods, and frequent use of ECPs is likely to lead to a higher incidence of side effects and a higher total hormone intake than other hormonal contraceptive methods. ${ }^{11}$ Furthermore, most contraceptive options cost less than ECPs (Research International/Ghana, personal communication, May 2008).

Given the drawbacks of ECPs and the availability of other options, it may be beneficial for some ECP users to switch to contraceptive methods that are more effective and possibly safer for long-term use - a transition known as 'bridging'. ${ }^{12}$ Successful bridging of ECP users to more effective contraception, however, is dependent on understanding the reasons women choose ECPs beyond use in emergency situations, and women's acceptance of a longer-term contraceptive method. The goal of this formative research study was to investigate the potential for bridging ECP users who purchase the product in pharmacies to longer-term contraception.

\section{Methods}

Data for the current study were collected in May 2008 in Accra, Ghana during the formative research phase of a larger intervention study. The intervention was designed to target ECP users in pharmacies, and transition them to OCPs through the provision of two free cycles of OCPs and an informational brochure that included a comparison of ECPs versus other contraceptive methods. The focus of this article is the formative research that looked at ECP users' contraceptive practices, feelings about ECPs and reasons for using ECPs instead of other methods. Additionally, participant reactions to the brochure messages were obtained.

Six top-selling pharmacies of ECPs agreed to participate in this study. Interviewers stationed at each participating pharmacy intercepted and recruited women purchasing ECPs, and asked them to participate in a semi-structured interview. Women were eligible to

Table 1 Characteristics of study participants

\begin{tabular}{llc}
\hline Characteristic & Category & Participants $(\boldsymbol{n})$ \\
\hline Age range (years) & $18-24$ & 9 \\
Marital status & $25-29$ & 15 \\
University status & Married & 4 \\
& Single & 20 \\
Use of ECPs in past 12 months & Student & 7 \\
& Non-student & 17 \\
& 1 time & 2 \\
& 2-3 times & 7 \\
& $4-6$ times & 5 \\
& $7+$ times & 8 \\
& Inconclusive & $2^{*}$ \\
\hline
\end{tabular}

*Two participants were unable to enumerate the number of times they had used ECPs.

ECPs, emergency contraceptive pills. participate if they reported buying ECPs that day for their own use and if they were aged between 18 and 30 years. In addition, minimum quotas were set to ensure a cross-section of participants based on age, marital status, student status and ECP use in the past year. In total, 24 ECP users, after having consented to participate, were interviewed one-on-one, during a 4-day period. Interviews lasted between 45 minutes and 1 hour.

Transcripts were audio-recorded, transcribed verbatim and finally coded and analysed using $\mathrm{NVivo}^{\mathrm{TM}}$ software Version 8 (QSR International, Doncaster, Victoria, Australia). The initial codebook followed the structure of the interview guide and was revised after reviewing several transcripts. ${ }^{13}$ Primary codes followed the structure of the interview and focused on the major research questions, while secondary codes were developed to cover information that arose extemporaneously. Two analysts performed periodic intercoder reliability checks to ensure consistency between coders. Three main topic areas were analysed: (1) ECP users' contraceptive practices; (2) ECP users' feelings about ECPs; and (3) ECP users' reasons for using ECPs over other methods.

\section{Results}

The average age of participants was 25 years, most were single and not attending university, and the majority reported using ECPs between three and six times in the last year (Table 1). There were no discernible differences in the data according to age, marital status, student status, or frequency of ECP use.

\section{Topic 1: ECP users' contraceptive practices}

ECPs occupied a central role in participants' contraceptive method mix. When asked, almost all participants agreed that ECPs were their main method of pregnancy prevention. However, some participants were unclear about what qualified as EC. Several women confused ECPs and abortifacients, and branded ECP labels such as Postinor-2 ${ }^{\circledR}$ (also referred to as 'P-2') were mentioned as well as medications used 'off-label' such as Primolut $\mathrm{N}^{\circledR}$ (also known as the 'N-Tablet' and indicated for treating a wide range of menstrual disorders).

When asked what women in their community use for pregnancy prevention, participants most frequently named EC, condoms and non-modern methods. Most participants said they knew about OCPs, although only four women reported ever using them. A number of women also referred to use of the sponge. Very few mentioned hormonal injections, implants or IUDs, and no one reported current use of these methods.

"In my community, as in my friends ... most of them use Postinor 2. They are not serious with condoms so they use Postinor 2." [Single 22-year-old]

"I'm only aware of the P2, Secure ${ }^{\circledR}$ [a brand of OCPs] and the condoms, because those are what I know people use in preventing pregnancy.” [Single 25-year-old] 
Menstrual cycle regulation (called 'safe days' or 'safe periods' by participants) was the most common non-modern method used. Although never explicitly defined by participants, it was implied to mean abstaining from sex during the days when women believed they were most likely to become pregnant. Other nonmodern methods mentioned included drinking water with milk/sugar/Milo ${ }^{\mathrm{TM}}$ (a milk beverage with chocolate and malt), drinking broken and ground glass mixed with alcohol and other beverages, taking anti-worming medications, withdrawal, or using herbal medications. Many of these methods were also reported as a means to terminate unwanted pregnancies, as alluded to previously, and some participants did not clearly state whether they were using these methods for pregnancy prevention or abortion.

"Most people in my community use withdrawal. They also add more sugar to very small water and drink it after sex; sometimes they add a lot of Milo to a little water milk and drink it. They also use condoms. Some people can take six or more tablets of paracetamol at once, they said it helps prevent pregnancy but I don't know for sure if it's true, though I see people do it. They also use dewormer[s], Don Simon (the alcoholic one), Postinor-2 and Secure [OCPs]." [Single 21-year-old student]

\section{Topic 2: Use of and feelings about ECPs}

Almost every participant was enthusiastic about ECPs, and women reported few disadvantages to using them. Although participants were familiar with the side effects - nausea, faintness and intermittent bleeding (reported by more than half the women questioned) - these did not appear troublesome enough to discontinue use. A number of participants reported experiencing no side effects at all from ECP use.

"I like the emergency contraception because it makes the blood flow. When you menstruate it gives you an easy flow." [Single 25-year-old]

"I feel normal [after taking ECPs]. I have my normal menstrual flow. I don't feel anything. It is good to me, that's why I don't want to change it." [Single 27-year-old student]

Participants also believed that ECPs were very safe and effective. They expressed great confidence that the pills would protect them from unwanted pregnancies with few physical repercussions. In addition, participants liked the convenience of ECPs because they are used after sex, only as needed, and can be used privately and without a partner's knowledge. In four cases, women secretly bought ECPs because they did not want their male partners to know.

"My husband, if you tell him to use condoms, you will pack your things and leave the room right now. That's why I use the P2 secretly." [Married 30-year-old]

There was also a sense that using ECPs provided a sense of relief.
"Do you know something? Any time I have sex with my husband, I use P2 afterwards, I feel good. I feel relieved." [Married 28-year-old student]

Women were asked for their thoughts on repeated use of ECPs, and the majority were unconcerned; most stated that they had not experienced any side effects from repeated use, nor did they anticipate experiencing any problems in the future.

"I have not experienced any side effects by using Postinor-2 and I don't think repeated use is going to be any problem. In fact, I don't see any problem in using it repeatedly. As for me, there is no way I would stop using Postinor."

[Single 23-year-old student]

However, a few participants thought repeated use may cause cancer, infertility, or other reproductive health sequelae in the long run.

\section{Topic 3: Reasons for using ECPs over other methods}

Nearly all participants expressed negative perceptions about other types of hormonal contraception. Legitimate procedural drawbacks or side effects were often cited, such as the fact that daily pills can be forgotten, condoms can disrupt sexual activity, and some methods cause weight gain.

"After taking the three-month injections three times, I realised that my menstrual cycle was getting [irregular] and a lot of other things like that and I was gaining weight. At first I was weighing sixty something and later I went up to eighty something and my guy asked me to stop it, so I stopped it for a while, then he said we should choose another way. That was when I went for the Postinor." [Single 21-year-old student]

Conversely, some perceptions were based on misinformation. For example, a few women believed that contraceptive devices can become displaced in the body, or that future fertility could be impaired.

"I was afraid because I didn't want the situation where in the future I may not be able to have children." [Single 28-year-old]

When asked about using daily OCPs, participants' responses were overwhelmingly negative. Women who had never used OCPs feared side effects because of friends' negative experiences. A few told stories of friends who, after years of OCP use, were unable to bear children. The few participants who had used OCPs in the past disliked the side effects and the annoyance of having to take a daily pill. Over half of the women said they were concerned they would forget to take a daily pill.

"It's not really the effectiveness of the Secure [OCPs] that I was worried about but the fact is that sometimes I have to go somewhere very early in the morning and when I leave the house I would forget to put the pills in my bag. That means that for that particular day I'll not take the pills and missing the pills can make you pregnant at any time." [Single 25-year-old] 
Although most participants had heard of OCPs, women who had not taken them previously showed little understanding of how to take the pills beyond knowledge of the daily regimen. In fact, participants had limited comprehension of other contraceptive methods: a few reported never having used a condom, and familiarity with hormonal methods besides pills, such as DMPA and implants, was low. When presented with health messages comparing ECPs to 'regular' contraceptive methods, participants were typically skeptical of the information.

\section{"Regular contraception is not better than emergency con- traception. With this I've tried it before so you can't tell me that regular contraception is better than emergency." [Single 28-year-old] \\ "What if you tell me that the side effects are few? I will still not be comforted with it because I trust only the Postinor-2.” [Single 23-year-old student]}

Views on the type of woman that 'regular' contraception is appropriate for were frequently mentioned as a deterrent to respondents' uptake of other hormonal methods. Half of the participants believed that only married women should use 'regular' contraception. These women believed that husbands should have a say in when and what type of contraception is used.

"The women who are most likely to go for the oral form of contraceptive are married women who have different partners, who have kids and don't want to have more kids." [Single 23-year-old student]

"A lady who would like [regular contraception] should be a married woman who is taking regular contraceptive pills. But for a young girl like me, I would only take emergency contraceptive when I need it." [Single 22-year-old]

"The implants, I heard married women take them. So as for now I am not married so I wouldn't want to try it." [Single 23-year-old]

The other half of the participants believed that 'regular' contraception is appropriate for both married and single women. Some of the single participants believed that any woman can use 'regular' contraceptive methods, but most stated that they personally do not and would not use 'regular', ongoing contraception.

"Singles go for it and married go for it. Women with children and those without children would go for it." [Single 21-year-old student]

'Single women can use 'regular' contraception, but I wouldn't use it." [Single 24-year-old]

Finally, ECPs were preferred to condoms among the study participants. Although some women mentioned using condoms, their preference for ECPs was clear. Most participants had used condoms in the past; however, a few women had never used condoms before and instead reported using ECPs as their first and only method of pregnancy prevention. According to most participants, male partners did not like having sex with condoms, and men often introduced their partners to ECPs.
Women mentioned their own dislike of condoms more often than they mentioned their partner's dislike. Half of the participants feared that condoms would break or burst during use. This fear was based on either personal experience with condom breakage or learnt from the experience of friends. The few participants who practised concurrent use of ECPs and condoms worried about sexually transmitted infections (STIs) or appreciated that condoms were less expensive than ECPs.

"The condoms are not reliable: they burst. They can burst at any time when the guy is using it, so when it happened like that and I noticed that I could easily get pregnant, then I went to do some inquires from my friends and noticed that I could use Postinor-2. So, since then whether it bursts or not I use it just in case." [Single 22-year-old]

\section{Discussion}

The contraceptive beliefs and practices of this small and self-selected sample of Ghanaian ECP users demonstrate a strong and consistent preference for EC. The women in this study were highly satisfied with EC, embraced the convenience and the ease of taking ECPs, and found the side effects tolerable. Non-modern family planning methods were still important to women in this study, while the use of modern pregnancy prevention methods was minimal. In general, women had limited knowledge of the modern contraceptive options available to them. This lack of knowledge is reflected in recent data from the Ghana Demographic and Health Survey (DHS): 2008 data showed that only $13.5 \%$ of women used a modern method for contraception, and that sexually active unmarried women were much more likely than married women to use non-modern methods. ${ }^{14}$ While ever use of ECPs at $2.9 \%$ was negligible as reported in the 2008 DHS, knowledge of ECPs was noteworthy at $35.4 \%$ and better known by Ghanaian women than the diaphragm, foam/jelly and the lactational amenorrhea method.

One reason that 'regular' methods were underutilised among the study sample is that participants held a number of misconceptions about the effectiveness of these methods, and expressed discomfort with anticipated side effects. Rumours prevailed that these methods fail easily, or cause severe side effects, and participants were hesitant to even say they would try these options. Even when provided with information on the advantages (including lower cost) and greater effectiveness of 'regular' methods, women in this study remained reluctant to entertain the notion of changing contraceptive methods and may have been unlikely to bridge as a result of the planned intervention. Indeed, most women had an "I have this friend" story, with tales ending in pregnancy, weight gain, forgotten pills and infertility. These findings from Ghanaian ECP users are seen in other countries too, where research shows that fear of side effects is a major barrier to use of hormonal contraception. ${ }^{15-17}$

Women's strong preference for ECPs, especially as a main method of pregnancy prevention, could be a 
concern in the family planning community. Policymakers, providers and even women themselves have expressed concern over potential physical harm from repeated use of ECPs, although there has been no evidence of harm caused by repeated use of postcoital hormonal contraception. ${ }^{18}$ In addition, because ECPs do not offer protection against STIs and HIV, it is possible that widespread availability of ECPs and use as a main method could deflect from condom use, including among those at risk of STIs, and thus have a negative impact on STI and HIV rates. While previous research has established that ECPs are safe and effective, even when used multiple times, ${ }^{9}$ little is known about the potential effect of more widespread ECP use on condom use for disease prevention. Also, because the large majority of women who were interviewed did not like condoms, and their partners did not like condoms, it is possible that men are encouraging the use of ECPs because they do not want to use condoms. Research with young people across several countries does suggest that some young men might prefer to use ECPs rather than condoms. ${ }^{19} 20$

There are limitations in this small, qualitative study of women purchasing ECPs through pharmacy outlets. The opinions and experiences expressed by participants may not be fully representative of other women in Africa, or even of other Ghanaian women. In addition to being a self-selected group, these women were already ECP users and, as such, may prefer ECPs over other methods. Since it was not within the scope of the larger study, we did not recruit a comparison group of women who purchased other contraceptive methods in pharmacies, but it should be recognised that those women may have expressed a preference for their purchased contraceptive methods as well. Accordingly, future studies in pharmacies should include users of other contraceptive methods. Additionally, it appeared that participants did not all understand the concept of 'emergency contraception' in the same way, which may affect how some of the results can be interpreted. In spite of these shortcomings, however, the themes across interviews were consistent - regardless of age, marital status, student status, or frequency of ECP use and indicate that this sample of Ghanaian ECP users needs more information about the range of contraceptive options before they would be amenable to adopting more effective longer-term methods. Nevertheless, general lessons can be gleaned from the interviews that have important implications for future ECP research.

After years of intense campaigning for increased access to EC, the fact that some women are aware of and using ECPs as needed is a victory. Results from this research suggest that a bridging intervention would require a more nuanced approach that also acknowledges that use of ECPs as a main or 'regular' method may be appropriate for some women. Indeed, the fact that ECPs were preferred because they are used after sex, as needed, and can be used without the partner's knowledge, should guide us in moving forward. This is true not only for effective bridging interventions, but with approaches in general that meet the reproductive needs of women and men in view of all the contraceptive options currently available.

Acknowledgements The authors thank Alex Banful at the Ghana Social Marketing Foundation and the Reproductive Health Unit of the Ghana Health Service for their support and guidance during the planning and implementation of the study.

Funding Support for this research was provided by the William and Flora Hewlett Foundation. The views expressed in this article do not necessarily reflect those of the Hewlett Foundation or of FHI.

\section{Competing interests None.}

Ethics approval The Institutional Review Board of FHI and the Ghana Health Service Research Ethics Committee approved this study.

Provenance and peer review Not commissioned; externally peer reviewed.

\section{References}

1 Maharaj P, Rogan M. Emergency contraception in South Africa: a literature review. Eur J Contracept Reprod Health Care 2008;13:351-361.

2 Yam EA, Gordon-Strachan G, McIntyre G, et al. Jamaican and Barbadian health care providers' knowledge, attitudes and practices regarding emergency contraceptive pills. Int Fam Plan Perspect 2007;33:160-167.

3 Moreau C, Bajos N, Trussell J. The impact of pharmacy access to emergency contraceptive pills in France. Contraception 2006;73:602-608.

4 Sørensen MB, Pedersen BL, Nyrnberg LE. Differences between users and non-users of emergency contraception after a recognized unprotected intercourse. Contraception 2000;62:1-3.

5 Young L, McCowan LM, Roberts HE, et al. Emergency contraception - why women don't use it. N Z Med J 1995;26:145-148.

6 World Health Organization. Emergency Contraception (Factsheet No. 244). 2005. http://www.who.int/mediacentre/ factsheets/fs244/en/ [accessed 23 October 2010].

7 Blair L. 'Morning after' scare: Postinor 2 abuse worries pharmacists. Jamaica Gleaner, 30 November 2003. http:// jamaica-gleaner.com/gleaner/20031130/lead/lead1.html [accessed 23 October 2010].

8 Mawathe A. Kenya concern over pill popping. BBC News, Nairobi, Kenya, 14 July 2009. http://news.bbc.co.uk/1/ hi/8145418.stm [accessed 23 October 2010].

9 World Health Organization (WHO). Emergency Contraception: A Guide for Service Delivery. Geneva, Switzerland: WHO, 1998.

10 Hatcher RA, Trussell J, Nelson AL, et al. Contraceptive Technology (19th revised edition). New York, NY: Contraceptive Technology Communications, 2007.

11 Grimes DA, Raymond EG. Emergency contraception. Ann Intern Med 2002;137:180-189.

12 Task Force on Post-Ovulatory Methods of Fertility Regulation. Efficacy and side effects of immediate postcoital levonorgestrel used repeatedly for contraception. Contraception 2000;61:303-308. 
13 Ulin PP, Robinson ET, Tolley EE. Qualitative Methods in Public Health: A Field Guide for Applied Research. San Francisco, CA: Jossey-Bass, 2004.

14 Ghana Statistical Service (GSS), Ghana Health Service (GHS), and ICF Macro. Ghana Demographic and Health Survey 2008. Accra, Ghana: GSS, GHS, and ICF Macro, 2009.

15 Frost JJ, Singh S, Finer LB. Factors associated with contraceptive use and nonuse, United States, 2004. Perspect Sex Reprod Health 2007;39:90-99.

16 Gilliam ML, Warden MM, Tapia B. Young Latinas recall contraceptive use before and after pregnancy: a focus group study. J Pediatr Adolesc Gynecol 2004;17:279-287.
17 Nettleman M, Brewer J, Ayoola A. Reasons for unprotected intercourse in adult women: a qualitative study. J Midwifery Womens Health 2007;52:148-152.

18 Halpern V, Raymond EG, Lopez LM. Repeated use of pre- and postcoital hormonal contraception for prevention of pregnancy. Cochrane Database Syst Rev 2010;1:CD007595.

19 Baiden F, Awini E, Clerk C. Perception of university students in Ghana about emergency contraception. Contraception 2002;66:23-26.

20 Harper C, Ellertson C. Knowledge and perceptions of emergency contraceptive pills among a college-age population: a qualitative approach. Fam Plann Perspect 1995;27:149-154. 